\begin{tabular}{c} 
International Journal of Advanced Astronomy, 5 (2) (2017) 83-89 \\
International Journal of Advanced Astronomy \\
SPC \\
Website: $\begin{array}{c}\text { www.sciencepubco.com/index.php/IJAA } \\
\text { doi: } 10.14419 / \text { ijaa. } v 5 i 2.8263 \\
\text { Research paper }\end{array}$ \\
\hline
\end{tabular}

\title{
Alternative explanation of some total solar eclipse related phenomena
}

\author{
Salah Abunaieb * \\ Sogexoman company, Muscat, Sultanate of Oman \\ *Corresponding author E-mail: salnayib@hotmail.com
}

\begin{abstract}
Diamond ring, shadow bands and coronal heating are some phenomena of great interest to concerned scientists and observers during total eclipse of the sun. These phenomena are explained here in a different way which is simply based on the familiar fact that the sun and the moon are actually spherical objects and not just plane disks. Firstly, the diamond of the diamond ring is seen here as two symmetrical seamless joined halves. One-half is the directly seen single spot of the sun that is uncovered by the moon just at the beginning and end of totality. The other half is a mirror image of that spot reflected by the edge surface of the moon. Secondly, the shadow bands phenomenon is explained as due to interference of light from two seamless joined hair crescents. One is the inner edge of the thin crescent sun that can be seen immediately before and after totality. Light from this inner edge hair crescent falling on the edge surface of the moon gets mirror reflected creating another hair crescent image and both are seamlessly embraced by the main crescent sun. Thirdly, during totality when looking at the outer atmosphere of the sun around its eclipsed face, one is actually looking through a very deep transparent atmosphere of the sun. The viewed depth in the outer atmosphere is geometrically hundreds of times greater than that of the photosphere at the center of the face of the sun. Theoretically, the solar energy gets concentrated by the integral effect of all the points along the viewed depth. This may, hence, stand as one of the factors to be considered in explaining the peculiar phenomenon that the temperature increases as we go away from the surface of the sun.
\end{abstract}

Keywords: Eclipse; Solar; Totality; Diamond Ring; Shadow Bands; Corona; Photosphere; Chromosphere.

\section{Introduction}

The idea behind writing this paper dates back to August 1999 while watching the live TV coverage of the total eclipse of the sun that happened by then. The TV coverage brought wonderful live sights of the different phases of the eclipse of the sun from different places around the world. The most spectacular sight to the author was the diamond ring that appeared just at the beginning and end of totality. Surprisingly, though the start of the eclipse was a small bite of the sun by the moon, the diamond of the diamond ring was a small bite of the dark face of the moon by the uncovered spot of the sun at that moment. To the author, the diamond of the diamond ring appeared as two symmetrical seamless joined halves. One-half was the directly seen uncovered single spot of the sun and the other was a mirror image of that spot reflected by the edge surface of the moon. It was particularly that event that was behind the different look which led to the alternative explanation, not only of the diamond ring, but also of shadow bands and coronal heating phenomena.

The TV coverage was also enriched by reports and interviews with concerned scientists and experts. From these reports and interviews, it was noted that, terms such as "Face" and "Disk" were frequently used without any conservation. Well, it was understood that these improper terms were only figuratively used to simplify explanation of the solar eclipse and its related phenomena. Nevertheless, such improper terms may sometimes influence concepts. So, looking at the sun and the moon as spherical objects viewed by an imaginary external observer is a basic idea in the following discussion.

\section{Diamond of the diamond ring is composed of two symmetrical halves}

The diamond ring effect occurs at the beginning and end of totality during the total solar eclipse. The effect happens for a very short period of time when a single bright spot of sunlight is uncovered by the moon. At that moment, our sky will become dark enough for the faint corona to shine out around the edge of the moon. The two effects together create the most striking sight known as the diamond ring [1], [2].

The alternative explanation given here is different from above explanation in only that the diamond of the diamond ring is actually composed of two symmetrical seamless joined halves. One-half is the directly seen single uncovered spot of the sun just at the beginning and end of totality. The other half is a mirror image of that single uncovered spot of the sun reflected by the edge surface of the moon. This is clear from the left hand side image shown in Figure 1 below (downloaded from the web site [3]). Now let us complete the disk of the moon by drawing a circle around its dark face as shown on the right-hand side of Figure 1. The circle cuts the diamond into two symmetrical halves. One is outside the circle which is the directly seen uncovered spot of the sun. The other is inside the circle which is supposed to be covered by the full circular face of the moon. So, this is clearly a mirror image of the outer uncovered spot of the sun. The inner half is too big to be considered as one of Baily's Beads plus it does not persist. 


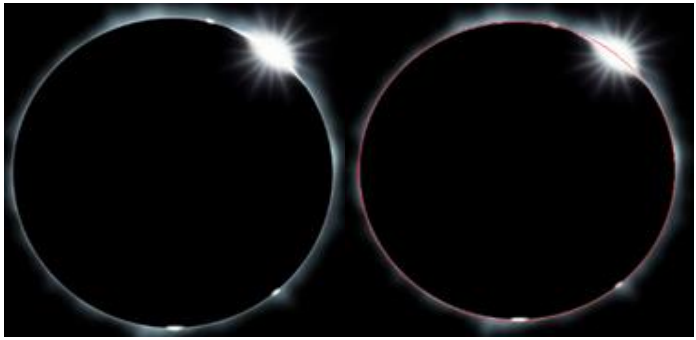

Fig. 1: Source: [3]

The idea that the edge surface of the moon is that reflective is supported by the following examples from our daily life observation. Relatively smooth surfaces can become mirror reflective when light from objects is falling with very large angles of incidence (close to $90^{\circ}$ ) or, in other words, when it falls almost parallel to the surface. A good example is the dry asphalt road. The dry asphalt road is relatively smooth, but it is not a surface that you can see your face in. Still, from certain distances and at certain places where the road is smoothly depressed or inclined one can see mirror image of low height objects, e.g. low height traffic sign boards, lower parts of vehicles on the road and low horizon as shown by photos in Fig. 2 and Fig. 3 below. Also, night time we can see images of head and rear lights of cars as shown in Fig. 4.

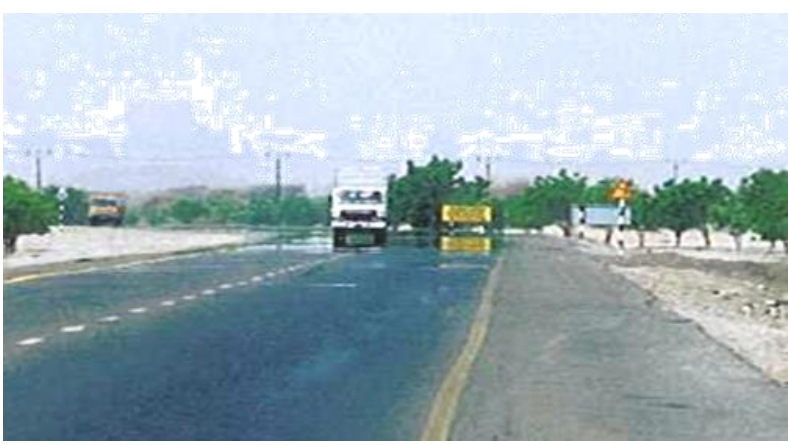

Fig. 2: (By Author).

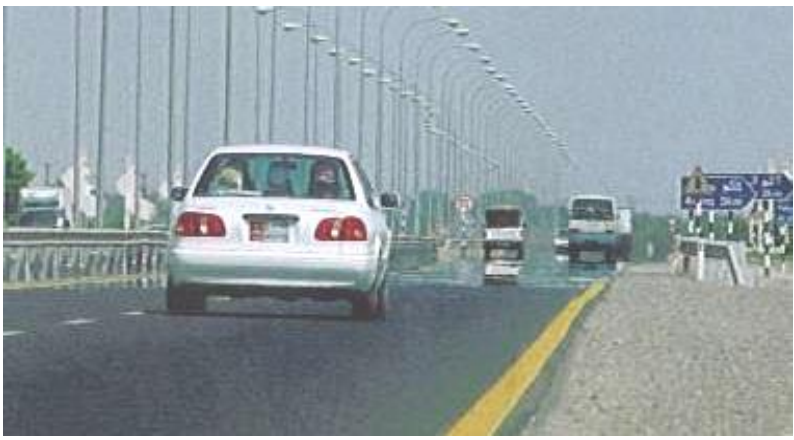

Fig. 3: (By Author).

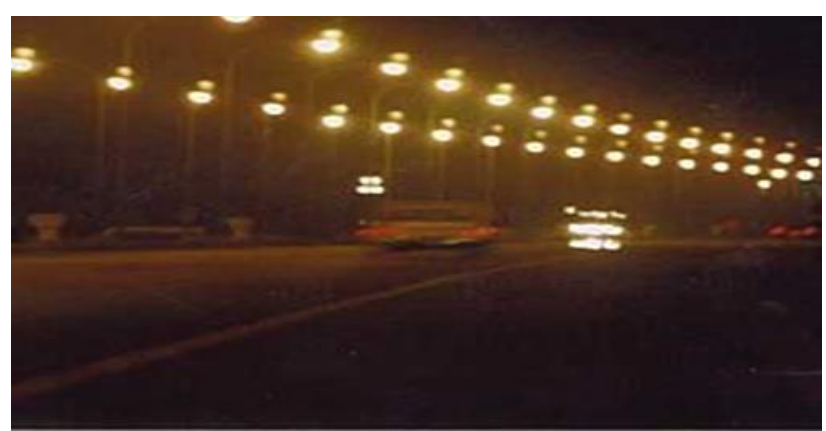

Fig. 4: (By Author).
This is different from images of high objects resulting from refraction of light during hot seasons. Images by refraction need no surface while the images shown above were on the dry surface of the road and not on the rough land around it. Also, the reflection by the dry surface of the road can be seen night time when it is cool. In the photo shown above in Fig. 4, you can see head lights of cars and their mirror images on the road. This while the street lights are not reflected at that point because of their height.

Now, going back to our basic idea, the moon is a sphere and its edge is hence a 3-dimensinally curved surface and not just a 2dimensionally curved line. Also, the moon is quite distant from earth and hence appears smooth. Light falling from the uncovered spot of the sun during the diamond ring effect falls almost parallel to the edge surface of the moon. Hence, like in the previous daily observed reflection examples, a mirror image of that spot is formed on the edge surface of the moon.

\section{Shadow bands phenomenon is due to inter- ference of light from two hair crescents}

The following present description and explanation of shadow bands phenomenon is quoted from Earth View Inc. webpage [4]: "While a small crescent of the sun remains in the sky, a curious eclipse phenomenon is often observed. Thin wavy lines of alternating light and dark can be seen moving and undulating in parallel on plain light-coloured surfaces. These so-called shadow bands are the result of sunlight being distorted by irregularities in the Earth's atmosphere, and are best observed on an open floor or wall.” The image shown in Fig. 5 below is from same webpage.

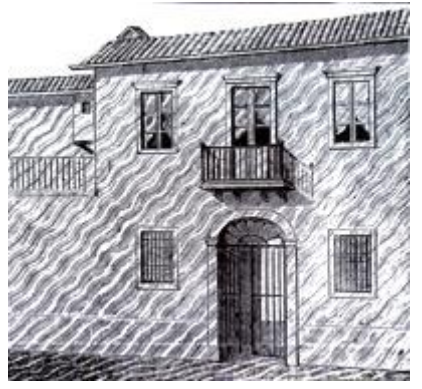

Fig. 5: Source: Earth View Inc. Webpage [4].

So, the above reference explains the phenomenon as due to distortion of sunlight by irregularities in our atmosphere. This while the description given in words, as quoted above, or by the image in Fig. 5 from the same reference show that the alternating light and dark lines are regularly wavy, parallel, spaced and of regular alternate thicknesses. Such regularity in pattern cannot be due to irregularities.

The alternative explanation given here is that shadow bands phenomenon is due to interference of light from two seamless joined hair crescents. One is the inner edge of the thin crescent sun that can be seen immediately before and after totality. This inner edge hair crescent is hereafter called the source hair crescent. Same like for the diamond ring, light from this source hair crescent falling almost parallel to the edge surface of the moon will get mirror reflected creating another hair crescent image which is hereafter called the reflected hair crescent. The reflected hair crescent is seamlessly embraced by the source hair crescent. Both hair crescents are in turn seamlessly embraced by the main crescent sun. So, they all appear as one whole crescent sun. As per the laws of reflection of light, the incident ray, the reflected ray and the normal to the reflecting surface at the point of incidence lie in the same plane [5]. But the normal at any point on the surface of a spherical body is radial. Hence the plane of the incident ray from any point on the source hair crescent and the reflected ray at the point of incidence on the edge surface of the moon will be radial with respect to the moon as shown exaggerated in Fig. 6 below. 
So like that when considering point by point the image of the convex side of the source hair crescent will be the concave side of the reflected hair crescent and the image of the concave side of the former will be the convex side of the latter as shown exaggerated in Fig. 6. The dashed circle is the assumed maximum height for the points to get mirror reflected.

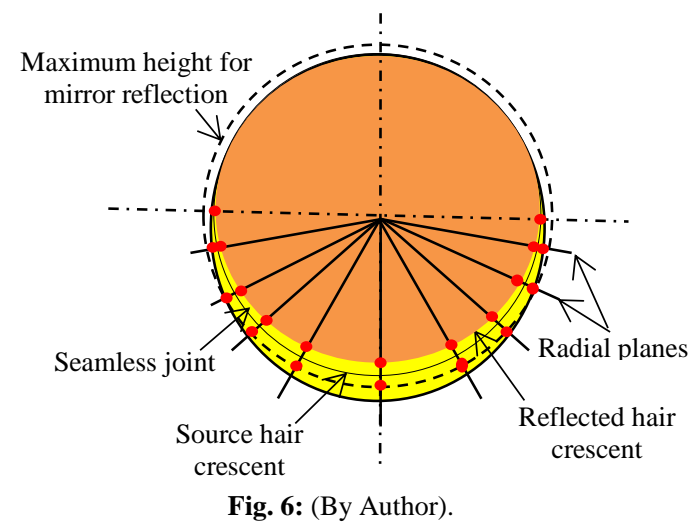

So, now except at the tips of the two horns of the whole crescent sun and on the seamless joint, each and every point on the source hair crescent will be seen as two points separated by a wedgeshaped space as shown below in Fig. 7. It is evident that the reflected light path of any given point on the source hair crescent is longer than its direct light path as shown below.

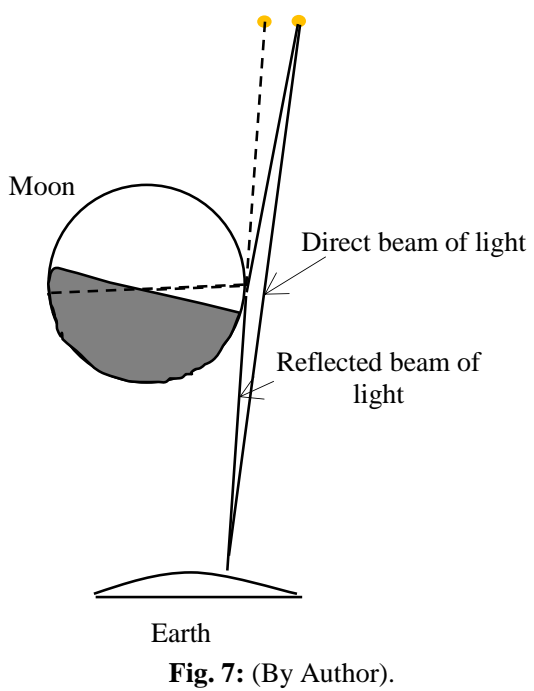

Same as in the Michelson interferometer according to Skinner [6], or as in the famous two-slit experiment according to Hawking [7], this difference in path lengths results in interference pattern. But in the Michelson interferometer all the mirrors and the ground glass in front of the extended source of light are plain. And in the two-slit experiment the partition between the light source and the screen is a plain partition with two narrow straight parallel slits. That is why in both the Michelson interferometer and the two-slit experiment the result of light interference is a characteristic pattern of straight light and dark fringes. But in the case of shadow bands the source of light and its reflected image are crescentshaped. That is why the fringes resulting from interference of light from the two crescents will, to some good extent, take the shape of a crescent but not exactly. In the Michelson interferometer if the movable mirror is displaced so that the difference in path lengths of the two beams of light changes by one wavelength of light, a new fringe will cross a given point on the field of fringes [6]. The difference in path lengths of a beam light from a point on the source hair crescent and the beam of light from its image is a function of the product of the direct light path length and the wedge angle. In equation form, Let $\mathrm{L}_{1}$ be the direct light path length, $\mathrm{L}_{2}$ the reflected light path length and $(\alpha)$ the wedge angle as shown below in Figure 8.The difference in path lengths $(\Delta \mathrm{L})$ is then simply related to the direct path length $\mathrm{L}_{1}$ and the wedge angle $(\alpha)$ as follows:

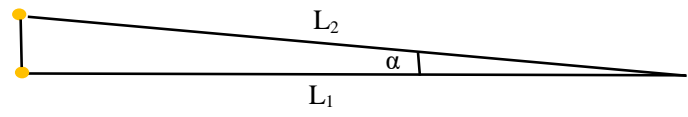

Fig. 8: (By Author).

$\Delta \mathrm{L}=\mathrm{L}_{2}-\mathrm{L}_{1}=\mathrm{L}_{1} \sec \alpha-\mathrm{L}_{1}=(\sec \alpha-1) \mathrm{L}_{1}$

From above equation, for a constant wedge angle the difference $(\Delta \mathrm{L})$ is directly proportional to the direct path length $\left(\mathrm{L}_{1}\right)$. And for a constant path length the difference $(\Delta \mathrm{L})$ is proportional to the wedge angle $(\alpha)$. Now let us see the effect of the two factors on the difference of path lengths of the direct and reflected light beams of the points along the two crescents. Bearing in mind the basic idea that the sun and the moon are spherical objects then it can easily be imagined that the mid-point of the two crescents is a little bit closer to the observer on earth than the two end points. Moving left or right from the mid-point towards the end points then the direct light path length gradually increases and hence the difference $(\Delta \mathrm{L})$. On the other hand and as roughly shown in Fig.6, the wedge angle for about two thirds of the distance from the midpoint towards the respective end point is almost constant. For the last third of the distance the angle gradually decreases till it reaches zero at the end point. Hence in the last third of the distance the gradual increase in $(\Delta \mathrm{L})$ due to gradual path length increase is accompanied by a gradual decrease due to decreasing angle. Accordingly if we put a crescent-shaped line on the field then the crescent fringes will deviate from that crescent line in one of two possible unit patterns as shown roughly below in Fig. 9 .

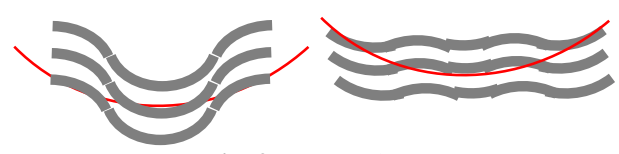

Fig. 9: (By Author).

Now for the full picture one would like to go back to childhood when the moon used to share playing one puzzling game with the children. In that game the children used to run in randomly different directions. Each child in turn used to suddenly change direction, speed or suddenly stop and restart while kept looking at the moon. The amazing result was that the moon was just following each and every child in whatever direction and at whatever speed exactly same like it was every child's own moon. When grown up we have come to know that it was the distance of the moon that was behind the amazing game. Of course the same is more accurately true about the sun which is many times more distant. So from any point in some normal human activity field the hair crescents can be seen the same way. Considering the distance of the sun and the distance and real size of the moon then one can imagine that there will be a vast surface on the edge of the moon for a wide range of intersecting reflective curves as shown in Fig. 10.

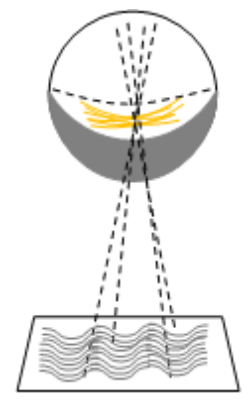

Fig. 10: (By Author). 
The combined unit patterns from the pairs of hair crescents related to each of the reflective curves make the total shadow bands pattern.

\section{Coronal heating phenomenon and the viewed depth in the solar atmosphere}

Since it was demonstrated in the 1940s, coronal heating has remained as one of the most puzzling problems in astrophysics. The temperature on the visible surface of the sun (photosphere) is around 6000 degrees Kelvin. Unexpectedly, going away from the surface the temperature increases. It reaches more than one million degrees Kelvin in the corona which is the outermost layer of the solar atmosphere that is normally visible during the total solar eclipse [8]. For the last more than seven decades, several different mechanisms have been proposed to explain how the heat is transported to the corona. But till date there is no one definitive answer for why the corona is 200 to 500 times hotter than the photosphere. Most of the researchers in this field believe that the answer is not just one definitive answer but a mix of different answers [9], [10]. As well, the View Concentration Ratio (VCR) presented here is no definitive answer. It is just one simple factor to be added to the pack of the several different explanations of this vexing phenomenon. The VCR is the ratio of the product of the viewed depth and the average energy flux throughout the viewed depth in the outer solar atmosphere to the product of the same along the depth of the photosphere at the centre of the solar disk. Bearing in mind our basic idea that the sun is a spherical object as viewed by an imaginary external observer, then we will find that the VCR is related to the way we view the eclipsed sun. Fig. 11 roughly illustrates the difference between the way we normally see the totally eclipsed sun (left-hand side hand side) and the way we see it in view of an imaginary external observer as shown sectionalized on the righthand side.

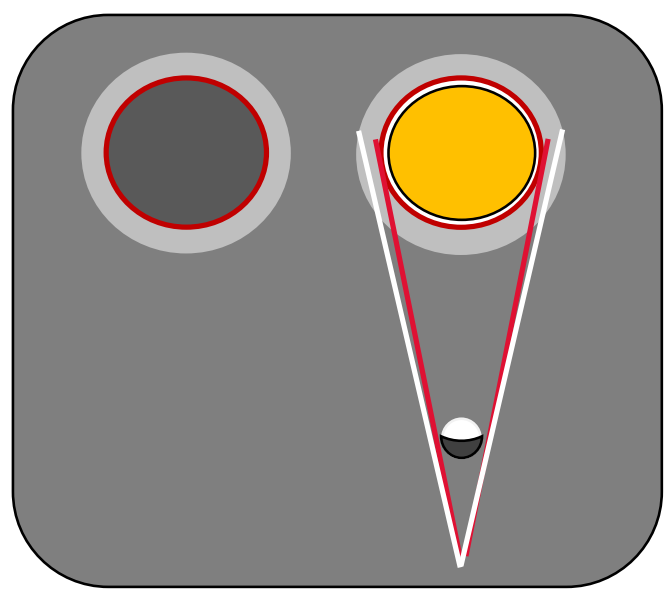

Fig. 11: (By Author)

On the left-hand side, the dark disk is the moon blocking out the bright visible face of the sun (photosphere). The reddish ring around the dark face of the moon is the chromosphere which is part of the outer solar atmosphere just atop the photosphere. The faint white outer ring is the corona which is the outermost layer of the solar atmosphere. These two layers of the solar atmosphere become visible during the total eclipse of the sun, but during normal times are not possible to see with the naked eye against the overwhelming brightness of the photosphere. On the right-hand side, the orange disk is the opaque sphere of the sun containing the core which is the nuclear reactor providing the solar energy by radiation and then by convection to the solar surface. The white ring around it represents a sectionalized view of the photosphere. The reddish and the outer faint white rings are sectionalized views of the chromosphere and the corona respectively.
So, as illustrated on the right-hand side of Fig. 11, when looking around the moon at any point on the surface of the outer atmosphere we are actually looking very deep into the optically very thin outer solar atmosphere. As it is physically connected to the surface of the sun, the outer atmosphere is considered here as a source of energy and not just a medium that scatters and absorbs some of the photons emitted from the photosphere. Every point in the solar atmosphere is considered as a source point of energy at some level. How much deep the line of sight goes into the outer atmosphere is a measure of the sum of energy of all the points, from the hottest point deep into the atmosphere to the coolest at the exterior surface, on that part of the line of sight. Hence came the term View Concentration Ratio (VCR)

However, the coronal heating phenomenon has not been found in that simple direct approach of energy concentration nor by direct temperature measurement. Coronal heating was concluded from some spectral lines in the spectrum of the corona which were found to originate from ions of heavy elements such as iron at very high stages of ionization, something that can only happen at very high temperatures [11]. Presently, space launched high tech instruments observe the solar atmosphere by tracking properties of material over a wide range of temperatures [12]. Furthermore, the solar atmosphere above the photosphere is known to be spatially highly non-uniform and very dynamic time wise. It consists of plasma (electrons, protons and ions) and is shaped by the structure of the magnetic field in the solar atmosphere. Shooting up jets of plasma, known as spicules, rise from the chromosphere and can extend up to $10,000 \mathrm{~km}$ into the corona. In the corona there are also regions which are hotter and denser than their surroundings contained in complex magnetic loop structures that are anchored in the photosphere [13], [14]

So, though the calculated VCR values for the selected range above the photosphere, as will be shown later, are found to match the ratio of the temperature in the corona to the temperature of the photosphere ((200 to 500), still, the VCR does not look suitable for explaining the coronal heating phenomenon in the highly active regions such as chromospheric network, spicules, flares and magnetic loops. It looks more suitable for explaining the phenomenon in the less dynamic and somewhat uniform balance of the outer atmosphere that constitutes by far the major part of the volume of the solar atmosphere.

To find out the VCR values at different heights above the photosphere let us first make the following assumptions:

1) As mentioned above, the highly active regions such as chromospheric network, spicules, flares, prominences, coronal holes, coronal loops and coronal mass ejections, are excluded. The VCR is limited to the bigger balance of the volume of the solar atmosphere which is assumed uniform and static for the selected range above the photosphere.

2) The solar energy flux ratio at any spherical level (or simply the energy level) in the outer solar atmosphere is the inverse ratio of the square of the radius of that sphere to the square of the radius of the top of the photosphere. The energy level is assumed unity throughout the photosphere. The VCR, as defined earlier, is the ratio of the product of the viewed depth and the average energy level along the viewed depth in the outer solar atmosphere to the product of the same along the depth of the photosphere at the centre of the solar disk.

3) Consequent on point 2 above, the paradoxical increase of temperature with height above the photosphere, which is normally observed from outside the solar atmosphere, is an integral energy effect of all the points along the viewed depth. The temperature of the individual elementary points, like in nature, decreases as we go away from the photosphere.

4) In agreement with the second law of thermodynamics (Holman [15]), the heat outward flow is from the highest temperature point at the maximum viewed depth to the lowest at the outer surface of the selected range of solar atmosphere. 
5) Since the chromosphere and the corona can be distinguishably observed during total eclipse of the sun, the VCR values are separately found for the chromosphere from just above the photosphere to near its maximum average height without considering the coronal layer above it. And for the corona from a little above the transition region (the thin layer between the chromosphere and the corona) up to a little below the selected maximum height. The transition region is assumed a gradual overlapping of the two distinguishable layers, and the VCR in this layer is only graphically found.

6) The lines of sight from the observer on earth to all the points within the selected range above the photosphere are assumed parallel.

7) The outer solar atmosphere is optically very thin and hence the viewed depth is only geometrically found.

Considering the above assumptions let us divide the outer solar atmosphere into spherical energy levels as shown below in Fig 12 .

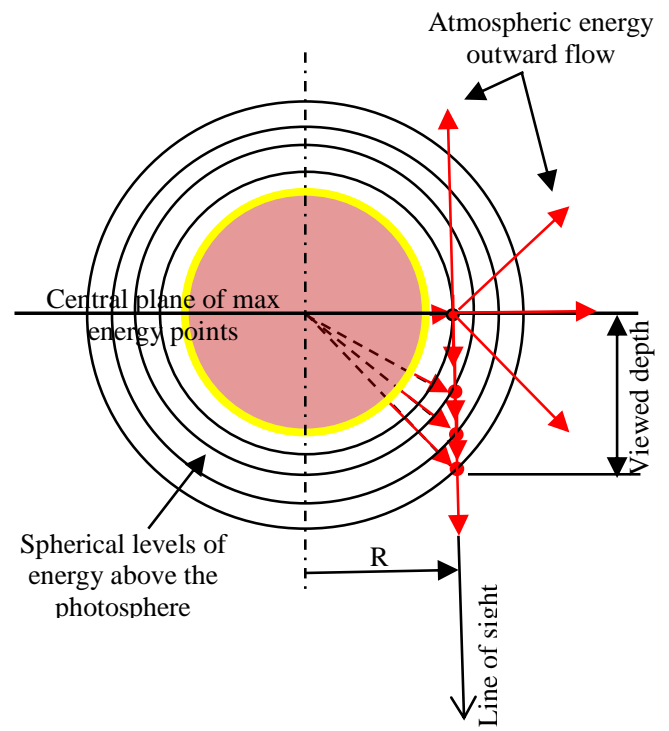

Fig. 12: (By Author)

Now let us consider looking at any point on the outer surface of the solar atmosphere at some radial distance $\mathrm{R}$ from the centre of the solar disk and hence through the atmospheric depth behind it. As shown in Fig. 12, the energy level gradually increases as the line of sight goes deeper into the solar atmosphere. It reaches its maximum energy level (the hottest) at the point where the line of sight becomes tangential to the spherical energy level of radius $R$ from the centre of the sun. Going further up beyond this point the energy level gradually drops. With the assumed parallel lines of sight, this point is on the central plane of maximum energy points on which all the parallel lines of sight have their respective hottest points. For the integral effect of energy along the viewed depth, we have to consider the direction of heat flow from the highest to the lowest temperature point, and hence the effective viewed depth is from the point on the outer surface to the central plane only. Another important note is that each point on the viewed depth (see Fig. 12) individually gets its respective energy level radially from the surface of the sun.

In equation form the energy level at any height $\mathrm{h}$ above the photosphere is as follows:

$\mathrm{E}=\mathrm{R}_{0}^{2} /\left(\mathrm{R}_{0}+\mathrm{h}\right)^{2}$

Where, É is the energy level as defined in above assumptions, $\mathrm{R}_{0}$ is the radial distance from the centre of the sun up to the top of the photosphere and $\mathrm{h}$ is any height above the photosphere.

From equation (2), the energy level versus height above the photosphere is shown graphically in Fig. 13 below.

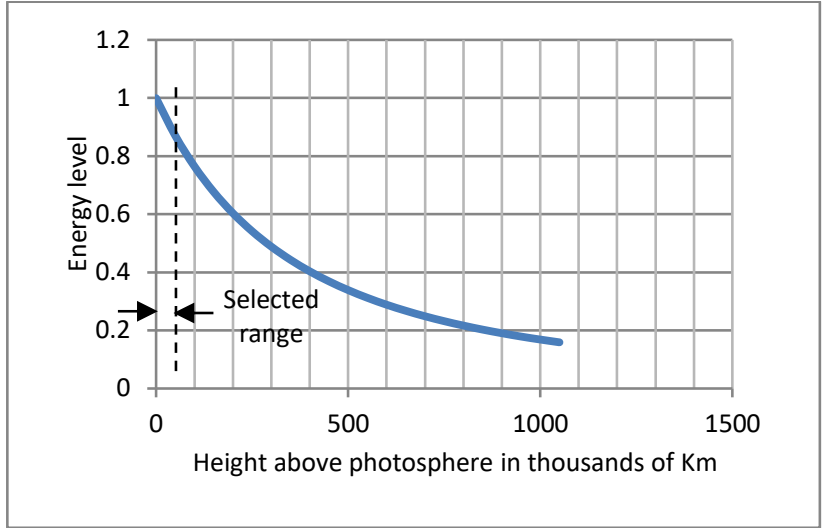

Fig. 13: (By Author).

As an inverse square function, the energy level drops with the height. The chromosphere has an average height of only around $2,000 \mathrm{Km}$ with very slight drop of energy level from 1 at the bottom to around 0.995 at the top of the chromosphere. But the corona is known to extend for many times the solar radius. At about only one and a half solar radius the energy level considerably drops to less than 0.200 .

However, considering the main objective of explaining the coronal heating phenomenon and for simplicity, the selected maximum height in the corona is limited to $50,000 \mathrm{Km}$ above the photosphere. In this range the energy level drops almost linearly from 0.995 at the top of the chromosphere to around 0.870 at the outer surface of the selected maximum range. So, the average energy level throughout any viewed depth in the chromosphere and within the selected coronal range can reasonably be taken as the arithmetic mean of the highest at the maximum viewed depth and the lowest energy level at the outer surface.

Now, from equation (2) and with the aid of Fig 14 below, the VCR in the chromosphere and the corona is simply found as follows:

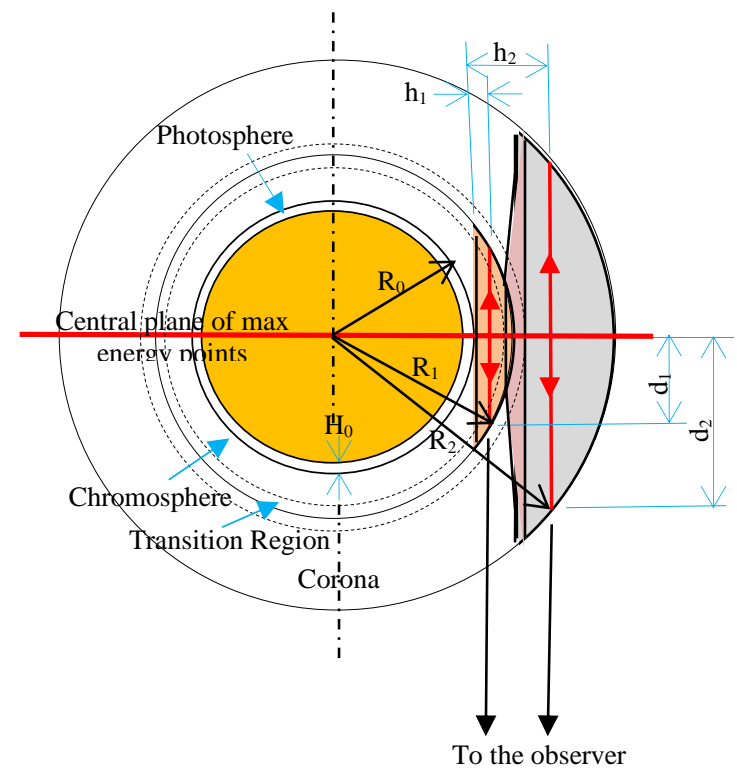

Fig. 14: (By Author).

1) In the chromosphere:

Viewed depth $(\mathrm{d})=\mathrm{d}_{1}=\left(\left(\mathrm{R}_{1}{ }^{2}-\left(\mathrm{R}_{0}+\mathrm{h}_{1}\right)^{2}\right)^{1 / 2}\right.$

Highest energy level $=E_{h}=R_{0}^{2} /\left(R_{0}+h_{1}\right)^{2}$

Lowest energy level $=E_{\mathrm{w}}=\mathrm{R}_{0}^{2} / \mathrm{R}_{1}^{2}$

Average energy level $=E_{\mathrm{a}}=\left(\hat{E}_{\mathrm{h}}+\hat{E}_{\mathrm{w}}\right) / 2$ 
$\mathrm{VCR}=\mathrm{d}_{1} * \mathrm{E}_{\mathrm{a}} / \mathrm{H}_{0}$

Where, $R_{0}$ is the radial distance from the centre of the sun up to the top of the photosphere and $R_{1}$ is the radial distance up to the top of the chromosphere, $h_{1}$ is any height $h$ above the photosphere and below the top of the chromosphere and $\mathrm{d}_{1}$ is the corresponding viewed depth $\mathrm{d}$ in the chromosphere. $\hat{E}_{\mathrm{h}}, \hat{E}_{\mathrm{w}}$ and $\dot{E}_{\mathrm{a}}$ are respectively the highest, the lowest and the average energy level throughout the viewed depth and $\mathrm{H}_{0}$ is the depth of the photosphere at the centre of the solar disk with an assumed average energy level of unity.

2) In the corona:

Viewed depth $(\mathrm{d})=\mathrm{d}_{2}=\left(\left(\mathrm{R}_{2}{ }^{2}-\left(\mathrm{R}_{0}+\mathrm{h}_{2}\right)^{2}\right)^{1 / 2}\right.$

Highest energy level $=E_{h}=R_{0}^{2} /\left(R_{0}+h_{2}\right)^{2}$

Lowest energy level $=\hat{E}_{\mathrm{w}}=\mathrm{R}_{0}^{2} / \mathrm{R}_{2}^{2}$

Average energy level $=E_{\mathrm{a}}=\left(\dot{E}_{\mathrm{h}}+\hat{E}_{\mathrm{w}}\right) / 2$

$\mathrm{VCR}=\mathrm{d}_{2} * \mathrm{E}_{\mathrm{a}} / \mathrm{H}_{0}$

Where, $\mathrm{R}_{2}$ is the radial distance up to the maximum selected height in the corona, $\mathrm{h}_{2}$ is any height $\mathrm{h}$ above the photosphere and below the maximum selected height and $\mathrm{d}_{2}$ is the corresponding viewed depth $d$ in the corona.

As can be noted from above equations, the two sets of equations are mainly different in the values of the constants $R_{1}$ and $R_{2}$. The VCR, the viewed depth $d$ and energy level versus height $h$ above the photosphere as shown below in Table 1 are found by substituting the height $\mathrm{h}$ and the following provided constants ([13], [16], [17]) in equations (3, 4, 5, 6 and 7) for the chromosphere and equations $(8,9,10,11$ and 12$)$ for selected range of the corona:

Radius of the opaque sphere of the sun $=696,265 \mathrm{Km}$.

Depth of the photosphere $=400 \mathrm{Km}$.

$\mathrm{R}_{0}=696,265+400=696,665 \mathrm{Km}$.

Depth of the chromosphere $=2,000 \mathrm{Km}$.

$\mathrm{R}_{1}=\mathrm{R}_{0}+2,000=696,665+2,000=698,665 \mathrm{Km}$.

Depth of transition region $=100 \mathrm{Km}$.

Selected maximum height of the corona as assumed above = $50,000 \mathrm{Km}$.

$\mathrm{R}_{2}=\mathrm{R}_{0}+50,000=696,665+50,000=746,665 \mathrm{Km}$.

Table 1: VCR, Viewed Depth and Energy Level vs. Height

\begin{tabular}{|c|c|c|c|c|c|c|}
\hline & $\mathrm{h}$ in $\mathrm{Km}$ & $\begin{array}{c}\mathrm{D} \text { in } 10^{\wedge} 3 \\
\mathrm{~km}\end{array}$ & $E_{\mathrm{h}}$ & $E_{w}$ & $E_{a}$ & VCR \\
\hline \multirow{4}{*}{$\begin{array}{l}\text { : } \\
\text { : }\end{array}$} & 0 & 52.827 & 1 & 0.994 & 0.9971 & 132 \\
\hline & 600 & 44.207 & 0.998 & 0.994 & 0.9963 & 110 \\
\hline & 1200 & 33.425 & 0.997 & 0.994 & 0.9954 & 83 \\
\hline & 1800 & 16.716 & 0.995 & 0.994 & 0.9946 & 42 \\
\hline \multirow{16}{*}{ రัँّ } & 2200 & 262.862 & 0.994 & 0.871 & 0.9321 & 613 \\
\hline & 3000 & 260.725 & 0.991 & 0.871 & 0.9310 & 607 \\
\hline & 4000 & 258.026 & 0.989 & 0.871 & 0.9296 & 600 \\
\hline & 6000 & 252.528 & 0.983 & 0.871 & 0.9268 & 585 \\
\hline & 8000 & 246.892 & 0.977 & 0.871 & 0.9240 & 570 \\
\hline & 10000 & 241.108 & 0.972 & 0.871 & 0.9212 & 555 \\
\hline & 12000 & 235.165 & 0.966 & 0.871 & 0.9185 & 540 \\
\hline & 14000 & 229.050 & 0.961 & 0.871 & 0.9158 & 524 \\
\hline & 16000 & 222.749 & 0.956 & 0.871 & 0.9131 & 508 \\
\hline & 18000 & 216.247 & 0.950 & 0.871 & 0.9104 & 492 \\
\hline & 20000 & 209.523 & 0.945 & 0.871 & 0.9078 & 475 \\
\hline & 24,000 & 195.322 & 0.935 & 0.871 & 0.9025 & 441 \\
\hline & 28,000 & 179.915 & 0.924 & 0.871 & 0.8974 & 404 \\
\hline & 36000 & 143.912 & 0.904 & 0.871 & 0.8873 & 319 \\
\hline & 40000 & 121.792 & 0.894 & 0.871 & 0.8825 & 269 \\
\hline & 46000 & 77.184 & 0.880 & 0.871 & 0.8753 & 169 \\
\hline
\end{tabular}

The VCR versus the height $h$ is shown graphically in Fig. 15 below. The graph is generated from Table 1 by taking the height $h$ values and the corresponding VCR values, continuously from 0.0 at the top of the photosphere to $20,000 \mathrm{Km}$ within the maximum selected range in the corona. The difference in the constants $\mathrm{R}_{1}$ and $\mathrm{R}_{2}$ is behind the horizontal shift of the curve in the chromosphere from that in the corona. In the transition region, which is assumed a gradual overlapping of the chromosphere and the corona, the maximum height is gradually increasing from $\mathrm{R}_{1}$ at near the top of the chromosphere to $\mathrm{R}_{2}$ at the bottom of the corona as shown above in Fig. 14. That is why the part of the curve in the transition region can be considered as a continuous series of points shifted horizontally from the maximum in the corona to the minimum point in the chromosphere.

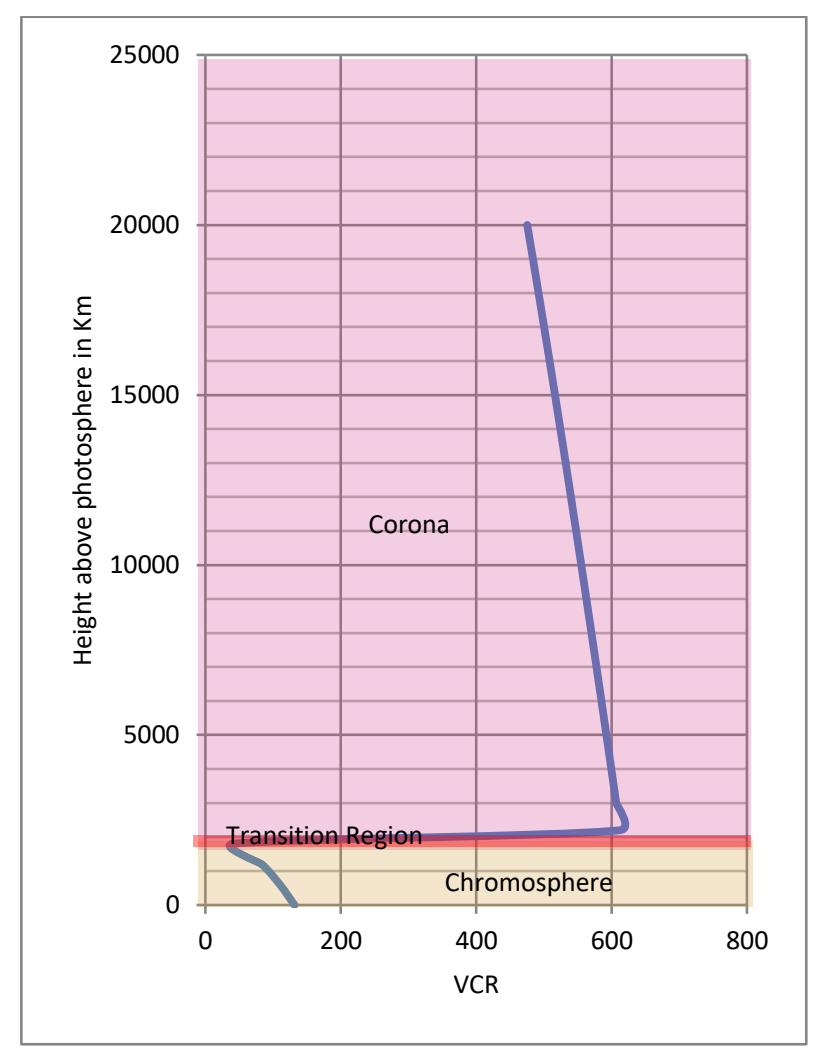

Fig. 15: (By Author).

Limiting the height $\mathrm{h}$ in Fig. 15 to $20,000 \mathrm{Km}$ only within the $50,000 \mathrm{Km}$ selected range is to allow comparison with the temperature and density versus height graph of Wikipedia contributors [14], shown below in Fig. 16.

Now taking some liberty of considering the VCR as a direct representation of the ratio of temperature in the outer solar atmosphere to the average temperature in the photosphere, let us compare the two graphs. In both graphs the highest rate of increase is within the thin transition region layer. In Fig. 16 the temperature (the solid white curve) increases very rapidly from about 10,000 at the bottom to about 1.0 million $\mathrm{K}$ at the top of the transition region. Then the temperature continues to gently increase in the corona reaching more than 1.0 million $\mathrm{K}$ at the height of $18,000 \mathrm{Km}$ above the photosphere. In Fig. 15 the VCR increases very rapidly from around 50 to around 500 within the thin transition layer. In the corona and at the height of $18,000 \mathrm{Km}$, the VCR is still at a high value of about 500. But unlike in Fig. 16, it reaches this value at a noticeable decreasing rate from about 600 at the bottom of the corona. This noticeable rate of decrease is because of the approximation made by limiting the corona to only $50,000 \mathrm{Km}$ range. Theoretically and as per equations $(8,9,10,11$ and 12), the VCR curve is going towards zero at the outer end of the selected range. But in actual fact we know that the corona extends for several million kilometres above the photosphere. So, if we select a very wide range with more accurate energy level values, the VCR 
curve in the corona for the compared range of height will then look flattened.

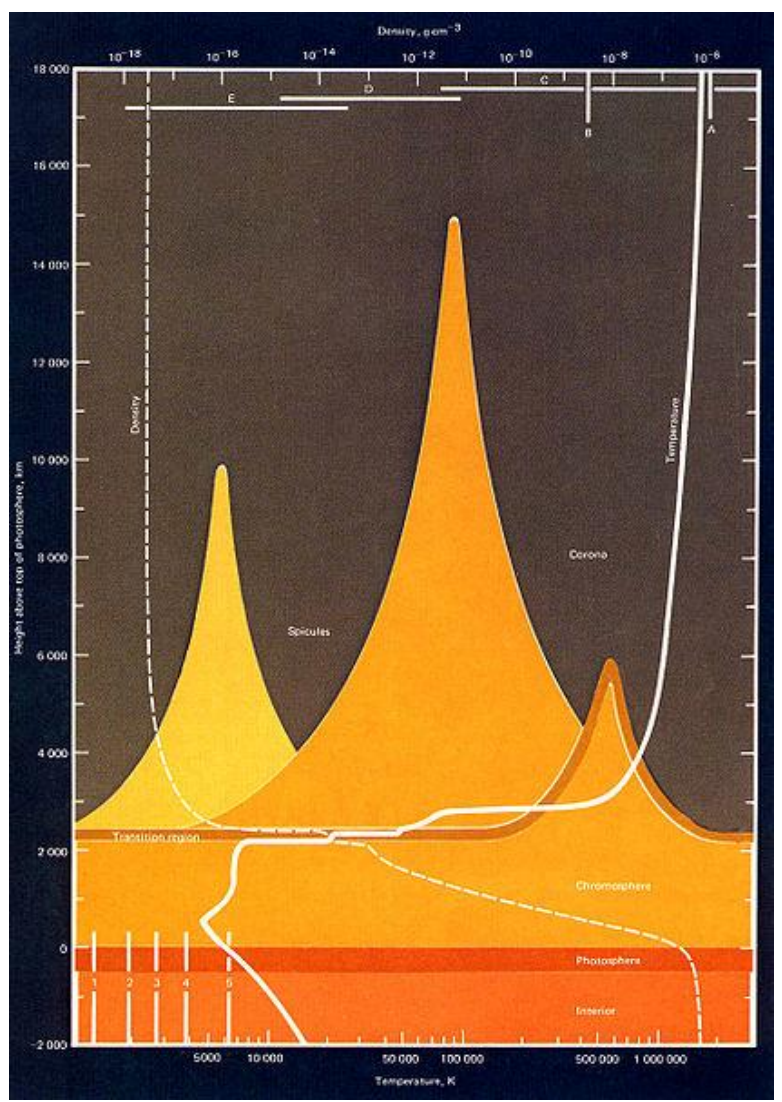

Fig. 16: Source: Wikipedia Contributors [14]

However, in the chromosphere the two graphs do not make good comparison. If we accept the VCR as a direct representation of the temperature ratio as mentioned above, then in the chromosphere the VCR values of more than 100 at the bottom to around 50 at the top are too big when compared to the ratio of temperature in the chromosphere to that in the photosphere. It seems that the assumption that the outer solar atmosphere is optically very thin is not reasonable for the chromosphere. The chromosphere is just atop the photosphere. The photosphere, though overwhelmingly bright, is known to be considerably opaque [18]. It seems that the effect of opacity of the photosphere, to some less extent, is affecting the chromosphere. So, generally speaking, opacity in the chromosphere will reduce the viewed depth and hence the VCR values versus height to some reasonable values. However, as mentioned earlier, the main objective of this study is to show the integral energy effect of the viewed depth as one of the explanations of the coronal heating phenomenon.

One last important note is about the non-uniformity of the outer solar atmosphere indicated roughly by the spicules rising from the chromosphere into the corona as shown in Fig. 16. It is interesting to note that the temperature curve trend does not seem to follow these changes in property of layer but rather follows the height.

\section{Conclusion}

Reflection of light by the edge surface of the moon when light is falling almost parallel to it gives good alternative explanation of both the diamond ring and shadow bands phenomena. The reflection phenomenon is supported by photos of similar daily life observation reflection examples. The most puzzling phenomenon, coronal heating, alternative explanation is excluding the highly active regions of the outer solar atmosphere. The paradoxical increase of temperature with height in the outer solar atmosphere as compared to the solar surface is explained as due to the integral effect of energy along the viewed depth through the optically very thin outer atmosphere of the sun. This explanation is found to be more appropriate in the corona than in the chromosphere. Being limited to the non-active balance of the volume of the solar atmosphere, this alternative explanation does not criticize nor does it stand as an alternative to any of the present explanations which are rather related to active regions of the solar atmosphere.

\section{References}

[1] Solar Eclipse Observing--The Diamond Ring and Baily's Beads. Excerpts from book by Dr. Michael D. Reynolds and Richard A. Sweetsir. Available at: http://www.alpostronomy.org/eclipse/observeeclipses/chapter9.htm. Accessed October 19, 2016

[2] Solar eclipse. WIKIPEDIA. Available at: https://en.wikipedia.org/wiki/Solar_eclipse. Accessed October 19, 2016.

[3] Diamond ring effect. Available at: http://www.weatherscapes.com/album.php?cat=astronomy\&subcas =diamond_ring. Accessed October 19, 2016.

[4] Effects during a Total Solar Eclipse. Available at: http//www.earthview.com/tutorial/effects.htm. Accessed August 02, 2016.

[5] Reflection (Physics). Available at: https://en.wikipedia.org/wiki/Reflection (physics). Accessed July 20, 2017.

[6] Skinner, R. (1982). Relativity for scientists and engineers. New York: Dover Publications.

[7] Hawking, S. (1995).A brief history of time. Toronto: Bantam Books.

[8] The way forward for coronal heating. Available at: http://astrogeo.oxfordjournals.org/content/49/3/3.21.full. Accessed July 23, 2016.

[9] The Mystery of Coronal Heating. Available at: https://science.nasa.gov/news-articles/the-mytery-of-coronalheating.Accessed July10, 2017.

[10] Physicists find new clue in coronal heating mystery. Available at: http://physicsworld.com/cws/artile/news/2011/jan/06/physicistsfind-new-clue-in-coronal-heating-mystery. Accessed July 01, 2017.

[11] The solar atmosphere. Available at: http://www.springer.com/9780-7923-7196-0. Accessed April 14, 2017.

[12] Best Evidence Yet For Coronal Heating, Theory Detected by NASA Sounding Rocket. Available at: https://www.nasa.gov/content/goddard/best-evidence-yet-forcoronal-heating-theory. Accessed July 01, 2017.

[13] The sun and the solar corona. Available at: www.sp.ph.imperial.ac.uk/ mkd/AndreHandout.pdf. Accessed April 14, 2017.

[14] Chromosphere. Available at: https://en.wikipedia.org/wiki/Chromosphere. Accessed April 18, 2017.

[15] Holman J.P. (1980).Thermodynamics 3rd edn. Mcgraw-hill Inc., US.

[16] Layers of the sun. Available at: www.nasa.gov/mission_pages/iris/multimedia/layerzoo.html. Accessed July 13, 2016.

[17] Sun. Available at: https://en.wikipedia.org/wiki/Sun. Accessed April 14, 2017).

[18] Sola Atmosphere. Available at: http://www.astro.cf.ac.uk/observatory/solarobservatory/background /?page=atmosphere. Accessed July 17, 2017. 